\title{
Application of Medical Big Data in Clinic
}

\author{
Jinguo Wang ${ }^{1, a}$ and corresponding author: Na Wang ${ }^{2, b,{ }^{*}}$ \\ ${ }^{1}$ Department of Urology, The First Hospital of Jilin University, Changchun, China \\ ${ }^{2}$ Department of Anesthesiology, The First Hospital of Jilin University, Changchun, China \\ awangjinguolily@163.com, bwangna080613@163.com
}

\section{Keywords: Application, Medicine, Big data, Clinic.}

Abstract. Main business application of big data is used to analyze the specific cases through health data model. Combined with the actual demand and development trend, the main business range of medical applications of large data includes intelligent auxiliary diagnosis, image data analysis, image intelligent diagnosis, rational drug use, remote monitoring, precise medical treatment, cost analysis, the effect of performance management, hospital fee and medical quality analysis. By medical data aggregation and circulation of platform, the big hospital and small and medium-sized hospitals through mutual referral, can share graded and medical treatment.

\section{Introduction}

Big data management platform is to establish standard system, provide an open uniform data, indicators caliber, interface visit, data security, trading and other specifications. Association of existing medical patterns provide effective resource sharing, the framework and platform construction specification, to make medical association platform to unify to improve the standardization of data and sharing. It provides decision support by using big data analysis and mining technology to integrate and analyze the resources of medical science. [1]

Data management of patients and hospital information is convenient, and implementation of medicine resources sharing of information is effective. [2] Through this platform, it provides an effective way to balance and optimize the medical resources of medical mediums, so as to facilitate the medical treatment of people and promote the development of medical association.

\section{Application of the Big Data in Clinic}

Rational Drug Use. The rational use of drugs is to choose the best drug pharmacology theory and its preparations, set or adjust the dosage regimen, according to disease type and the situation, for safe, effective and economical prevention and cure of diseases.

In addition to the implementation of national drug policy, standardizing medical behavior and strengthening measures are the effective measures to improve the level of rational drug use. Through the clinical rational drug use audit and consulting system, clinical physicians' medical behavior also can be standardized. [3]

By using big data technology, on the basis of patients' medical history, disease diagnosis, the doctor's advice, medication information, allergies and drug safety warning, such as drug taboos on drug interaction, compatibility taboo review, doctors can find unreasonable drug problem in a timely manner. In addition to the hospital prescription history data in data mining, analysis of antimicrobials, injection percent of prescription drugs, basic drugs, examining the fraction defective is issued by the hospital prescription and can provide data support for regulating medical behavior. [4]

Medical Quality Analysis. Medical quality analysis is the most important criterion to evaluate the overall level of hospital medical service and management, and has been the core of hospital management. [5] Using big data analysis technology, the quality of medical data is converted to managers need information index, according to the characteristics, historical data, charts, information, etc., to provide data support and basis for the management. It is an important application of medical and big data. 
In addition, the application of medical big data will be more and more extensive in the prediction of disease trend, health assessment, patient demand and behavior analysis, data analysis and electrocardiogram diagnosis. [6] Patient- doctor order data are similar to the data of medical records. The medical information platform also implements similar query and synchronization for the patient-doctor order data. Medical information platform is for all data recorded the whole life cycle of patients, because it is convenient to be used in medical personnel through the PC and mobile medical applications to track orders way of execution, the execution time, the time for observation on patient's illness, observation data real-time acquisition entry.

Precise Medical Treatment. Accurate medical large data analysis can accurately find the pathogenic causes, form accurate clinical diagnosis report, provide the best treatment plan for patients and minimize maximum therapeutic effect and side effects, by collecting personal complete clinical diagnosis and treatment records, electronic medical record system diseases with similar clinical diagnosis and treatment records, and connecting with the patients' genetic information, using bioinformatics analysis tools, ontology, data mining of data collected for big data analysis techniques such as integrated analysis. [7]

Provide reliable data for nursing work, and also regulate nurses' nursing work. Opened for patients with inspection examination data: the doctor inspection and check the application information, and test results, reports, and check the information in a structured form of document storage on medical information platform. The medical staff can check the progress and results of the application on the platform through the PC side application and mobile medical application. [8]

Remote Monitoring System. Remote patient monitoring system includes household heart test equipment, blood glucose meter, chip tablet, etc. [9] Remote monitoring system contains a lot of medical data. It can be related to patients symptoms in the remote monitoring system of data collection, through the analysis of the results after feedback to monitor equipment, data collection around the signs. It can analyze and judge the corresponding variation rule, combined with the history data and determine the medication and treatment in the future. At the same time, it can reduce the patient's hospital stay and alleviate the short of wards. [10]

Of hospital medicine proportion, outpatient medical costs, hospital patients per capita medical expenses, outpatient medical expenses growth, inpatient medical expenses per capita growth, typical single diseases patients with all fees, ginseng protect personal spending rate, medicare catalog costs, inspection and testing accounted for the proportion of medical income, medical health materials accounted for revenue share, registered/diagnosis/beds/treatment/surgery/nursing total revenue accounted for the proportion of medical income, one hundred yuan medical health materials fee, management fee income consumption, asset-liability ratio is analyzed.

\section{Mobile and Remote Medical System}

The main items include drugs, inspection, inspection, operation, nursing and treatment of categories. Besides, the cost data also includes materials, bed, nursing and replacement expenses. In smart handheld devices, mobile medical system, either as staff as the main body in the operation, such as for physicians clinical ward round the doctor diagnosis and treatment of mobile station, mobile nurse workstation for medical care, mobile outpatient transfusion application system, mobile drug management system, etc., as a main body or in the patients with operation, such as make an appointment, the medical registration, capture expends pay, medical appointments, health education, such as a mobile application, all need to hospital existing electronic medical records, electronic medical advice, medical management, pharmacy pharmacy information system for data exchange and business cooperation. [10]

Data of patient's medical records: medical information platform collects patient's medical records and documents in the form of documents structured in electronic medical records. Provide data for mobile applications to electronic medical records. [11] It also implemented the PC side application and 
mobile medical application to send messages to each other through medical information platform and provide data for synchronization after modification of medical records. [12]

Information collection and analysis system of big data will help patients find the nearest clinic medical institutions, intelligent classification diagnosis and treatment, by data mining algorithms to construct the intelligent diagnosis system. Patients with partial data will be uploaded to the big data acquisition platform. Scientific research activities can provide important reference for diagnosis and treatment. Intelligent diagnosis system and the data expert database will help make collaboration mechanism of association of medical hospital and attract patients diagnosis and classification, in patients with design framework. [13]

\section{Conclusions}

On the base of the requirement of system and function orientation, a large data of medical service system should be built from three aspects, including the medical information collection and sharing, data mining and knowledge management, individual medical service innovation. On the base of the theory of semantic web and ontology, data mining technology and the design of medical cloud put forward the training of the system.

During the realization of system, three aspects are discussed, including the semantic network, data mining and analysis, personalized service. The implementation of the mechanism shows the service content and process of the system.

\section{References}

[1] Lei Shi, Ying-Jie Han, Xiao-Guang Ding, Lin Wei, Zhi-Min Gu. An SPN-Based Integrated Model for Web Prefetching and Caching[J]. Journal of Computer Science and Technology. 2006 (4)

[2] Grobelnik Marko. Big Data Tutorial. http://videolectures.net/eswc2012_grobelnik_big_data/. 2012

[3] Thusoo A, Sarma J S, Jain N. Hive - a petabyte scale data warehouse using Hadoop. Proceedings of the 2010IEEE 26th International Conference on Data Engineering (ICDE 10). 2010

[4] Allison L Holloway, David J. DeWitt. Read-Optimized Databases In Depth. The VLDB Journal. August 24-30, 2008

[5] Jeffrey Dean, Sanjay Ghemawat. MapReduce[J]. Communications of the ACM. 2008 (1)

[6] David Martin, Mark Burstein, Drew McDermott, Sheila McIlraith. Bringing Semantics to Web Services with OWL-S[J]. World Wide Web. 2007 (3)

[7] Gate A F, Natkovich O, Chopra S. Building a high-level dataflow system on top of map-reduce: the pig experience. VLDB. 2009 (9)

[8] Anirban Mahanti, Derek Eager, Carey Williamson. Temporal locality and its impact on Web proxy cache performance[J]. Performance Evaluation. 2000 (2)

[9] Sanjay Ghemawat, Howard Gobioff, Shun-Tak Leung. The Google file system[J]. ACM SIGOPS Operating Systems Review. 2003 (5)

[10] Arun Venkataramani, Praveen Yalagandula, Ravindranath Kokku, Sadia Sharif, Mike Dahlin. The potential costs and benefits of long-term prefetching for content distribution[J]. Computer Communications. 2002 (4)

[11] Oracle Inc.Oracle database10g Oracle Data Guard: http://www.oracle.com/technology/deploy/availability/htdocs/DataGuardOverview.html. 2011

[12] Sara Cohen, Carmel Domshlak, Naama Zwerdling. On ranking techniques for desktop search[J]. ACM Transactions on Information Systems (TOIS). 2008 (2) 
[13] A. Pavlo, E. Paulson, A. Rasin. A Comparison of approaches to large-scale data analysis. Proc of the 2009 ACM SIGMOD Int Conf on Management of Data. 2009 